RESEARCH ARTICLE

\title{
Effects of an Intense One-Week Skiing Program on Balance Abili- ties in Pediatric Cancer Patients
}

\author{
Kurpiers $\mathrm{N}^{1^{*}}$, Vogler $\mathrm{T}^{2}$ and Flohr $\mathrm{S}^{1,3}$ \\ ${ }^{1}$ Department of Sport Science, University of Hildesheim, Germany \\ ${ }^{2}$ Center for Sports Medicine, Münster, Germany \\ ${ }^{3}$ Department of Biology, University of Hildesheim, Germany
}

*Corresponding author: Nicolas Kurpiers, PhD, Department of Sports Science, University of Hildesheim, Universitätsplatz 1, 31141 Hildesheim, Germany, Tel: +49-5121-883-11918

\begin{abstract}
Beside a number of physical, physiological, psychological, and social impairments, cancer and its treatment can also reduce balance abilities. The aim of the present study was to assess the effect of a one-week winter camp with an intense alpine skiing program on balance ability of pediatric cancer patients in the follow-up care. Eighteen pediatric cancer patients (intervention group IG; age $10.2 \pm 4.6$ years) and eight healthy children for comparison (healthy group HG; age $12.3 \pm 3.3$ years) participated in the intervention. The results were compared to corresponding readings of a control group that consisted of 13 pediatric cancer patients (control group CG; age $9.8 \pm 2.4$ years) of a conventional stationary rehabilitation program. Balance ability was tested by using a Nintendo Balance Board (NBB) equipped with a "Low Cost Force Lab" software as the measurement device, with the Mean Euclidian Distance as a measure for stability within a $30 \mathrm{sec}$ trial on both legs and on each leg separately. The results revealed that (a) the patients of the IG exhibited significantly lower balance abilities in the pretest in the one-leg trial for the left leg compared to the CG and the HG, (b) the IG improved their abilities significantly on both single-leg and the both-leg trial, and (c) that the IG improved their values close to that of the HG. The study showed that impaired balance abilities in pediatric cancer patients caused by the disease and its treatment can be improved within a rather short time period to the level of their healthy counterparts.
\end{abstract}

\section{Keywords}

Acute Lymphoblastic Leukemia, Postural Stability, Rehabilitation, Sports Therapy, Winter Sports

\section{Introduction}

Cancer is one of the main causes of death in the world [1]. With 144 to 148 cases per million, cancer prevalence is much smaller in children than in adults, with Acute Lymphoblastic Leukemia (ALL) being the most frequent entity in childhood cancer [2]. In both, children and adults, treatment of cancer can produce a number of severe side effects, depending on the type of cancer and the kind of therapy [2]. Cytostatic drugs, for example, often cause impairment of the bone marrow functions, whereas immunological cancer therapies frequently lead to irritations of the mucous membranes and a reduced immune defense [3]. Other side effects concern the physical fitness of the patients. In children suffering ALL, a reduced aerobic capacity was observed [4] as well as neuromuscular deficits depending on the medication dose [5]. Other authors documented sensory and motor impairments and neurologic signs that persisted up to five years after therapy in childhood ALL patients [6,7].

Until the 1980's, physical activity was considered contra-productive in cancer treatment and resting was a common medical advice. Influenced by the seminal study of Schüle [8] and based on a number of more recent clinical studies, this view has changed, and today physical activity and sports are generally considered to cause various positive effects in cancer patients, including psycho-social, physical, and immunological parameters and their interactions [9-15]. Positive effects of physical activity on cancer concern prevention, rehabil-

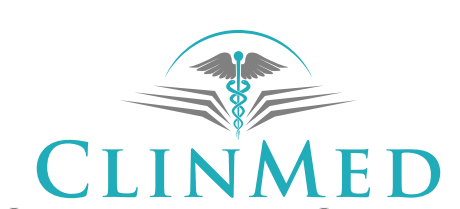

INTERNATIONAL LIBRARY 
itation, and relapse prevention. Regarding prevention, some studies suggest that physical activity might have a protective effect on breast cancer, colon cancer, and cancer of the endometrium $[9,16]$. The mechanisms behind this postulated effect are, however, poorly understood. The contribution of physical activity to rehabilitation is presumably the most intensively studied aspect, comprising a large amount of potential aims. They range from rather "mechanical" issues such as the increase of the range of motion after breast cancer surgery [17], the improvement of the reduced physical strength and endurance after cancer treatment $[18,19]$, to psycho-social effects that focus on the regaining of self-confidence, the avoidance or reduction of social isolation due to the disease [20], and the improvement of immunological parameters [21-23]. The effects of sports on relapse prevention are largely unknown since systematic studies on this issue are rare. However, a reduced risk of breast cancer recurrence has been reported in women with a physically more active lifestyle [24]. Advances in the diagnosis and treatment of cancer have led to higher survival rates and longer post diagnosis intervals and will probably lead to more research on life style effects on cancer recurrence, including the effects of physical activity and sports [24].

Most studies on the effects of physical activity on cancer have been conducted on adults. Much less research has been done in pediatric cancer patients [25]. Nevertheless, the studies conducted so far on the latter group are promising. Related to the prevalence of the cancer type, most of these studies were conducted on children suffering from ALL during rehabilitation $[4,17,26,27]$. Parameters tested in these studies comprise cardiorespiratory capacity, bone mineral density, flexibility, and aspects of health-related quality of life [2]. However, the reported effects of physical exercise on pediatric cancer patients were considered as not fully convincing, mainly due to the small sample sizes and methodological problems [2].

Motor skills in children develop rapidly. Any interruption of this development, e.g. in the course of a cancer disease and its treatment, can lead to a retardation in a number of these skills [28]. This can be accompanied by psycho-social consequences in affected individuals when interacting with other members of their age group. A decrease of balance ability is assumed to be one side effect of childhood cancer, depending on the cancer type, the treatment, and the time elapsed since treatment $[27,28]$. Balance is defined as the ability to maintain the body's center of mass within the base of support [7,29]. It is one of the most important motor skills since it is needed in almost every activity of daily life. However, systematic studies on balance abilities in pediatric cancer patients are rare, especially with respect to physical activity in rehabilitation $[27,30]$.

The aim of the present study was to investigate the effect of a one-week winter sports camp with an intense skiing program on pediatric cancer patients. The effects were compared with corresponding measurements in healthy controls that also participated on the winter sport camp and on pediatric cancer patients who participated in "regular" pediatric cancer rehabilitation programs. The study was part of a larger joint project between the University of Hildesheim, the Medical University Hannover, and the Pediatric Clinic Bad Oexen (all Germany).

\section{Methods}

A total of 31 pediatric cancer patients and eight healthy children (HG) participated in the study. Of the 31 cancer patients, 18 children formed the intervention group (IG; 9 boys and 9 girls) with an average age of 10.2 \pm 4.6 years (mean $\pm S D$ ). Most of the children of the IG had suffered from and been treated for leukemia ( $N=$ 13), the entities of the other children were brain tumor $(\mathrm{N}=2)$, bone tumor $(\mathrm{N}=2)$, and lymph gland tumor $(\mathrm{N}=$ 1). Patients of the IG were out of therapy between six and 60 months (on average 24 months). The control group (CG) consisted of 13 childhood cancer survivors ( 7 boys and 6 girls) with an average age of $9.6 \pm 2.1$ years. As in the IG, leukemia was the most frequent entity $(N=10)$. One girl suffered from brain tumor, one boy from tumor of the parotid gland, and one girl from myelodysplastic syndrome. The time interval since termination of cancer therapy varied between three and 31 months (average 14.5 months) in this group. Side effects of the cancer therapy were expressed in various ways and severities in the members of both groups, ranging from minor disturbances in coordination, initial signs of fatigue syndrome to temporary loss of sense of vision and hearing in one child. The HG consisted of eight children ( 5 boys and 3 girls) with an average age of $12.3 \pm 3.3$ years, most of them being siblings of the children of the IG.

Most patients of the IG and the HG were participants of one out of four one-week ski camps, conducted in March of the years 2015 to 2018. Two children (both of the IG) participated in two camps. In these children, only the data obtained from their first camp were considered for the present study. Within the camps, the children were guided in groups of up to five members by experienced guides who were familiar with the individual health related situation of the children. Thus, the guides were able to adopt the duration and intensity of the activities and exercises to the specific resilience of the group members. The groups consisted of children of both, the IG and the $H G$, i.e. the participants within a group followed the same training program. The groups were skiing twice a day for about two hours each, performing skiing-specific technical exercises and downhill runs. The first comprised basic exercises of the "one-ski-method" [31], with the number of runs increasing slowly following the improved fitness and skill level of the group members. The guides were free to organize the training program spontaneously according to the current situation.

Members of the CG were embedded in regular 
phy-siotherapeutic activities, i.e., they underwent a stationary rehabilitation including two to three sport or movement therapy programs per week. The programs comprised different physical activities such as ball games, climbing, horse riding or swimming. The duration of the sessions was about 90 minutes each. Again, and according to the common practice of the therapy center, the therapists were free to shape the training program. Since the goals of the regular therapy were of a rather general nature, a focus on specific motor skills was not given.

Balance abilities were tested twice, in the IG und the HG first at the beginning of the six days (pre-test) of intervention, and second on the last day of the intervention (post-test). In the CG, the test was performed in the same time interval in the course of the regular rehabilitation program.

The tests were conducted by using a Wii Nintendo Balance Board ${ }^{\circledR}$ (NBB) (Model RVL-021, Kyoto, Japan) equipped with a "Low Cost Force Lab" software (Biovision, Version 2015a, Wehrheim, Germany) as the measurement device. The NBB offers a simple platform with four measuring sensors and can detect, save and analyze the vertical ground reaction forces of the NBB with smoothed $100 \mathrm{~Hz}$ signals. The device was positively evaluated regarding its reliability and considered being appropriate to measure standing balance in clinical settings [32]. The NBB was connected to a laptop via Bluetooth to visualize the performance. The visualization consisted of a red dot on the screen which marked the center of pressure and the challenge for the test persons was to keep the point in position for 30 seconds. Each leg was evaluated separately as well as both legs together. The system calculated the Mean Euclidean Distance to the center of pressure. Thus, the smaller the value the more stable was the trial.

All participants and their parents were informed about the aims of the study and everyone was free to quit the participation at any time without justification. The ethics committees of the Hannover Medical School (for the IG) and of the University of Hildesheim (for the CG) gave approval, and the parents of the participants signed informed consent prior to the first test.

Comparisons between the groups (IG, CG, and HG) were calculated by one-way ANOVA with LSD (least significant difference) post-hoc test. Comparison of the results of pre-test and post-test as well as comparisons between the results of the right and the left leg were analyzed with a paired $T$-test, respectively. Because several $T$-tests were calculated on the data, Bonferroni adjustment of $P$-values was performed $\left(P_{a d j}\right)$. Resulting $P_{a d j}$-values $>1.0$ were rounded down to 1.0 [33]. $P$-values lower than 0.05 were considered significant. The statistical analyses were conducted using the software package IBM SPSS Statistics V23. For paired $T$-tests, the effect size was calculated by using the software GPower v3.1.9.2.

\section{Results}

As expected, the results of the two-leg trials were

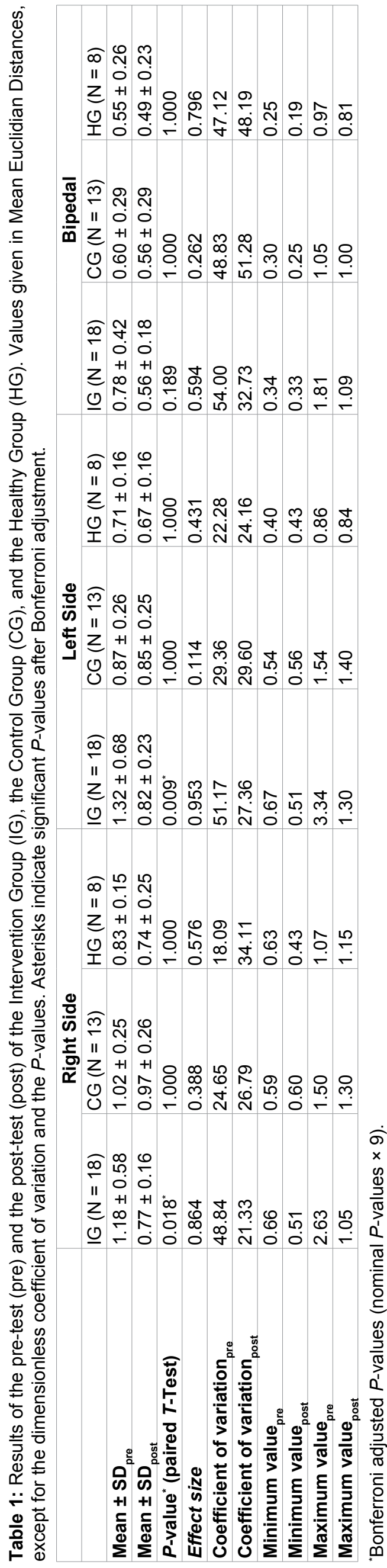


better than those of the one-leg trials (Table 1). Although the IG showed on average markedly higher values (i.e. less stable trials) on the both-leg trials in the pre-test than the other groups, the difference was not statistically significant ( $P=0.212$; one-way ANOVA). All groups showed better result in the both-leg trials in the post-test than in the pre-test, with all three groups exhibiting very similar values in the post-test. Improvement of balance (decrease of Mean Euclidean Distance) from the pre-test to the post-test was highest in the IG, although statistically not significant after Bonferroni adjustment ( $\mathrm{P}_{\text {adj. }}=0.189$; paired $T$-test; effect size 0.594).

Much larger differences as in the both-leg trials were measured in the single-leg trials. Here, the mean values of the IG showed the highest Mean Euclidian Distances (poorest balance ability), the HG the lowest (best balance ability; Figure 1) in both, pre-test and post-test. Concerning the differences between the groups in the pre-test, one-way ANOVA revealed significant differences for the left leg trials $(P=0.008)$. Here, the post-hoc test indicated significant differences between the IG and the CG $(P=0.016)$ with better results for the CG, and between the IG and the HG $(P=0.006)$ with better results for the HG (Figure 2).

As in the both-leg trials, an improvement of balance ability in the post-test compared to the pre-test was recorded for the single-leg trials in all groups. However, balance abilities in the CG and the HG improved only slightly and non-significantly $\left(P_{a d j .}>0.05\right)$, whereas the improvement in the IG was significant for both single-leg trials $\left(P_{a d j}=0.018\right.$; effect size 0.864 for the right side, $P_{a d j}=$ 0.009; effect size 0.953 for the left side; paired $T$-test), thereby leading to values close to those measured in the HG and better than those of the CG (Figure 1). Differenc- es in the post-test between the groups were found for the right leg-trials ( $P=0.024$; one-way ANOVA) resulting from significant differences between the IG and the CG ( $P=0.016$; post-hoc test), and between the CG and the HG $(P=0.023$; post-hoc test $)$.

The marked decrease in the Coefficient of Variation (CV) between the pre-test and the post-test in the IG in all trials including the both-leg trial (Table 1), reflects the homogenization of the performance in the children of the IG. In the other two groups, the CV differed only slightly between the pre-test and the post-test.

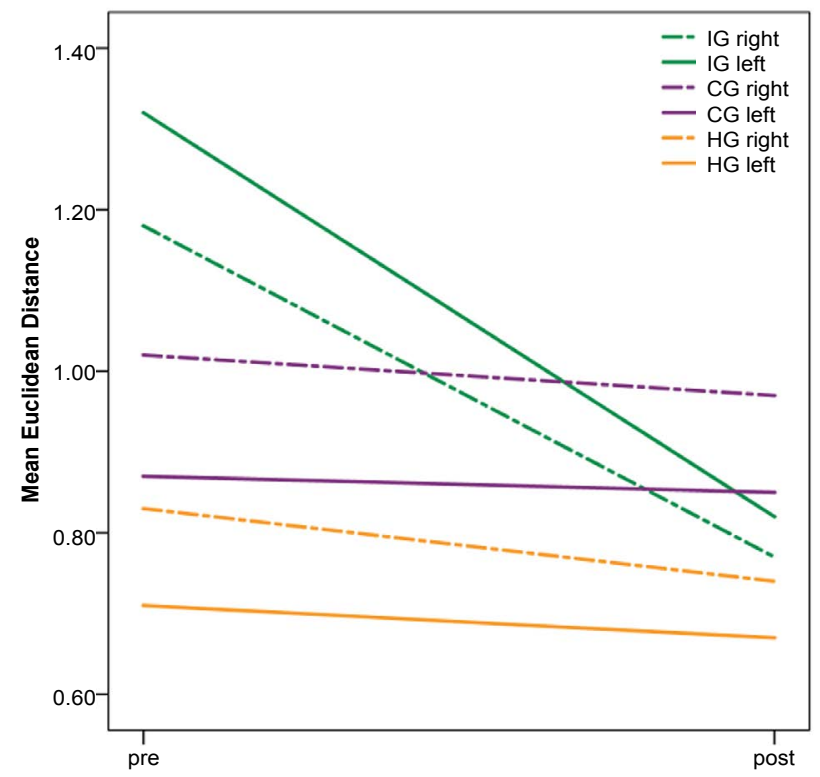

Figure 1: Results of the single-leg trials. Comparison of the average Mean Euclidean Distance for the groups in the pre-test and the post-test. Note that the values of the IG in the post-test are close to that of the HG.
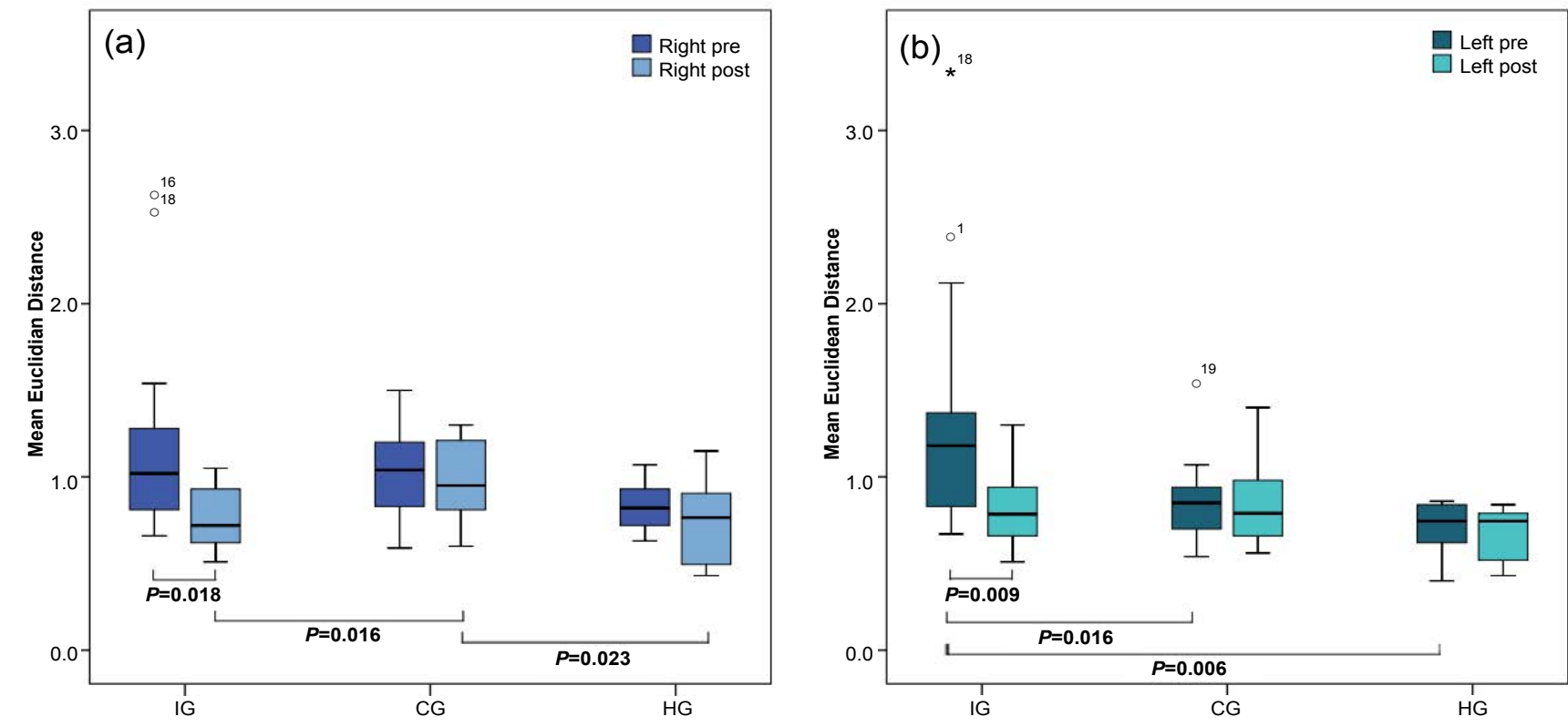

Figure 2: Results of the single-leg trials. The box plots show the results of the right leg (a) and the left leg (b) trials. Significant differences between groups are indicated (intra-group comparison: $T$-test with adjusted $P$-values; inter-group comparison: ANOVA with $P$-values of the LSD post-hoc test). 
Table 2: Comparison between right and left side in pre-test and post-test in the Intervention Group (IG), the Control Group (CG), and the healthy control group (HG). Paired $T$-test with Bonferroni adjusted $P$-values.

\begin{tabular}{|l|l|l|l|l|l|}
\hline & $\boldsymbol{N}$ & Right Side & Left Side & $\boldsymbol{P}$-values & Effect size \\
\hline $\mathrm{IG}_{\text {pre }}$ & 18 & $1.18 \pm 0.58$ & $1.32 \pm 0.68$ & 1.000 & 0.326 \\
\hline $\mathrm{IG}_{\text {post }}$ & 18 & $0.77 \pm 0.16$ & $0.82 \pm 0.23$ & 1.000 & 0.280 \\
\hline $\mathrm{CG}_{\text {pre }}$ & 13 & $1.02 \pm 0.25$ & $0.87 \pm 0.26$ & 0.204 & 0.668 \\
\hline $\mathrm{CG}_{\text {post }}$ & 13 & $0.97 \pm 0.26$ & $0.85 \pm 0.25$ & 0.930 & 0.429 \\
\hline $\mathrm{HG}_{\text {pre }}$ & 8 & $0.83 \pm 0.15$ & $0.71 \pm 0.16$ & 0.354 & 0.781 \\
\hline $\mathrm{HG}_{\text {post }}$ & 8 & $0.74 \pm 0.25$ & $0.67 \pm 0.16$ & 1.000 & 0.250 \\
\hline
\end{tabular}

*Bonferroni adjusted $P$-values (nominal $P$-values $\times 6$ ).

In the pre-test and in the post-test, the results of the right-leg trials were slightly better than those of the leftleg trials in the CG and the HG. In the IG, lower values were obtained in the left-leg trials. Regarding this symmetry of the trials, however, no significant differences were found between the sides within the groups (all $P_{a d j}$-values $>0.05$; paired $T$-test; Table 2).

\section{Discussion}

The aim of the present study was to investigate the effect of intense alpine skiing on balance abilities in pediatric cancer patients. The study showed that (a) pediatric cancer patients that were not included in systematic rehabilitation programs (here: IG) showed the poorest balance ability prior to the intervention, (b) that pediatric cancer patients that participate in a systematic rehabilitation program (here: $\mathrm{CG}$ ) show better but still reduced balance abilities compared to healthy controls (here: $\mathrm{HG}$ ). The study further showed that the members of the IG improved their balance abilities significantly within a rather short time period to values close to that of their healthy counterparts (HG) and better than the values of the members of the CG. No negative effects of the intense training on the young cancer patient were observed during the intervention.

For a patient, fighting against cancer means not only to overcome the disease itself but also to cope with the partly severe side effects of the therapy. There is growing evidence that physical activity can provide positive effects for the patient on several aspects associated with the disease, its sequels, and the side effects of the therapy. For example, the weakened muscles can successfully be strengthened [34-36], and positive effects on the fatigue syndrome, a common side effect in cancer therapy, are reported [37]. Reduced balance ability is another common side effect in pediatric cancer patients, largely independent of the kind of treatment (surgery, chemotherapy, radiotherapy) [30]. Gait disturbances were for instance found in pediatric patients surviving posterior fossa brain tumor surgery [38]. Balance and postural stability are results of a complex interaction between the visual, somatosensory, and vestibular systems [39]. Cancer and its treatments can affect one or several of these systems. It was for example reported that 30 to $40 \%$ of the patients treated with chemothera- py developed a peripheral neuropathy as one of the side effects [40]. Neurogenic impairments, either centrally or peripherally, might influence the proprioceptive system and thus lead to a reduced balance ability $[29,41]$.

Reduced motor skill abilities, including balance abilities, in pediatric cancer patients do not only result from the disease and its treatment but might also, to a certain degree, be associated with physical inactivity and sedentary behavior during and after the treatment [42-46]. This may partly reflect the obsolete view that resting is the first choice in curing cancer. However, only little is known about the effect of physical activity on balance abilities in pediatric cancer patients.

A number of studies showed positive effects of skiing on several motor skills, including balance performance [47], in healthy [48] and diseased adult individuals [49]. The latter study investigated elder patients suffering sarcopenia and concluded that skiing is an effective intervention to combat the disorder and weakness in these patients. Numerous other positive effects resulting from snow sport interventions, including self-esteem, standing balance and gross motor function [50], were also observed in people with disabilities. The latter authors emphasized further that the risk of injury was not higher in those groups than in the general population [50].

A major component in alpine skiing is the need for control of balance in a highly dynamic system. Each turn is characterized by a shift of the center of mass outside the base of support (imbalance) to initiate the change of direction of the run with a subsequent rapid instauration of a stable system in which the center of mass projects on the base of support (balance) [51]. It is therefore assumed that skiing is highly effective to increase reduced balance abilities. The results of the present study confirmed this view by showing a rapid and significant increase of balance ability in pediatric cancer patients.

It was beyond the scope of the study to detect the specific component(s) of the complex ability "balance" that contributed to the observed improvements. For example, on the basis of the present data it is unclear whether the increase of stability was the result of increased muscle strength caused by the intervention program or due to other factors. Muscle weakness has been stated as a common side effect of cancer treatment [27] and might be one common reason for reduced balance abilities in pediatric cancer patients $[28,30]$. Accordingly, a correlation between muscle strength and balance ability has been reported in ALL long-term survivors who showed impaired knee extension strength associated with two times poorer balance scores [27]. To detect the effect of skiing on specific components of "balance" (e.g. muscle strength, neurogenic factors, self-confidence), a much more sophisticated analysis of the participants' motor skills is required. However, such decided analyses were not performed for the present study. The study was rather part of a larger project in which 
pediatric cancer patients together with their parents and siblings were removed from the severely exhausting clinical settings into an environment that allows these families temporarily to get rid of the burdens that are associated to a cancer disease in a family member. We therefore tried to hold the amount of diagnostic procedures as small as possible for the children.

As many other studies, the rather small number of participants is a limitation of the study. The present study though contributes to the understanding of the potential role of physical activity in specific settings in pediatric cancer therapy, a largely underexplored field in sport science and the results are promising.

\section{Conclusions}

Physical activity in general has the potential to promote both, physiological parameters and health-related life quality parameters in cancer survivors [26]. Based on its biomechanical characteristics, alpine skiing was considered being an appropriate intervention with a high specificity regarding balance and postural stability to compensate reduced balance abilities in pediatric cancer patients. The present study demonstrated that alpine skiing indeed can compensate deficiencies in balance abilities in pediatric cancer patients within a rather short time period with almost reaching the level of their healthy counterparts. A direct comparison to the member of the CG, however, is difficult since the amount of training hours was much larger in our IG with about four hours skiing per day compared to two to three hours sports per week in the CG. The different changes in the balance ability between the groups might therefore also be related to the training frequency but we assume that the high specificity of alpine skiing has clear advantages to compensate deficiencies in balance abilities than general rehabilitation programs. The study demonstrated not only that a significant improvement of the reduced balance ability is possible but also that such an intense training is possible to perform with those children without any acute risks. Further studies should proof the sustainability of the present results and could also evaluate possible long-term effects on health-related parameters, including the recurrence of cancer.

\section{Acknowledgements}

We thank all funding bodies, participants, physicians, and guides that accompany this project. We also thank Konstantin Krauth, head of the children hospital Bad Oexen for his cooperation in the study, and the anonymous reviewers for their helpful comments on the initial draft of the manuscript.

This project was funded by the Lotto-Sport-Stiftung Niedersachsen, the Inner Wheel Club Hildesheim, the Vor-Tour der Hoffnung e.V., Menschen für Kinder e.V., and a number of private donators.

The Local Ethical Committees of the University of Hildesheim and the Medical University Hannover gave the approval. Informed consent was obtained from the participants and their parents in accordance with the Declaration of Helsinki.

The experiments comply with the current laws of Germany. The authors have no conflict of interest to declare.

\section{References}

1. Steward BW, Kleihues P (2003) World Cancer Report. World Health Organization: Geneva.

2. Braam $\mathrm{KI}$, van der Torre $\mathrm{P}$, Takken $\mathrm{T}$, Veening MA, van Dulmen-den Broeder E, et al. (2016) Physical exercise training interventions for children and young adults during and after treatment for childhood cancer. Cochrane Database Syst Rev.

3. Pasquali S, Mocellin S (2010) The anticancer face of interferon alpha (IFN-alpha): from biology to clinical results, with a focus on melanoma. Curr Med Chem 17: 3327-3336.

4. San Juan AF, Chamorro-Viña C, Maté-Muñoz JL, Fernández del Valle M, Cardona C, et al. (2008) Functional capacity of children with leukemia. Int J Sports Med 29: 163-167.

5. Ness KK, Hudson MM, Pui CH, Green DM, Krull KR, et al. (2012) Neuromuscular impairments in adult survivors of childhood acute lymphoblastic leukemia. Cancer 118: 828-838.

6. Green JL, Knight SJ, McCarthy M, De Luca CR (2013) Motor functioning during and following treatment with chemotherapy for pediatric acute lymphoblastic leukemia. Pediatr Blood Cancer 60: 1261-1266.

7. Lehtinen SS, Huuskonen UE, Harila-Saari AH, Tolonen U, Vainionpää LK, et al. (2002) Motor nervous system impairment persists in long-term survivors of childhood acute lymphoblastic leukemia. Cancer 94: 2466-2473.

8. Schüle K (1983) Zum Stellenwert der Sport-und Bewegungstherapie bei Patientinnen mit Brust-oder Unterleibskrebs. Rehabilitation (Stuttg) 22: 36-39.

9. Baumann FT, Jäger E, Bloch W (2012) Sport und körperliche Aktivität in der Onkologie. Berlin: Springer.

10. Courneya KS, Keats MR, Turner AR (2000) Physical exercise and quality of life in cancer patients following high dose chemotherapy and autologous bone marrow transplantation. Psychooncology 9: 127-136.

11. Courneya KS, Segal RJ, Mackey JR, Gelmon K, Reid RD, et al. (2007) Effects of aerobic and resistance exercise in breast cancer patients receiving adjuvant chemotherapy: a multicenter randomized controlled trial. J Clin Oncol 25: 4396-4404.

12. Schmelzle M, Schwarz R, Fellhauer S, Schlag P (1991) Zum Stellenwert von Nachsorgekuren in der Krankheitsbewältigung von Tumorpatienten. Onkologie 14: 61-65.

13. Schmitz KH, Courneya KS, Matthews C, Demark-Wahnefried W, Galvão DA, et al. (2010) American College of Sports Medicine roundtable on exercise guidelines for cancer survivors. Med Sci Sports Exerc 42: 1409-1426.

14. Schwibbe G (1991) Veränderungen der Lebensqualität onkologischer Patienten im Verlauf einer stationären Nachsorgekur. Rehabilitation 30: 55-62.

15. Wiskemann J, Huber G (2008) Physical exercise as adjuvant therapy for patients undergoing hematopoietic stem cell transplantation. Bone Marrow Transplant 41: 321-329.

16. Rank MV Freiberger, Halle M (2012) Sporttherapie bei Krebserkrankungen. Schattauer Verlag. 
17. Wilson DJ (2017) Exercise for the Patient after Breast Cancer Surgery. Semin Oncol Nurs 33: 98-105.

18. Hayes SC, Spence RR, Galvão DA, Newton RU (2009) Australian Association for Exercise and Sport Science position stand: optimising cancer outcomes through exercise. J Sci Med Sport 12: 428-434.

19. Knols R, Aaronson NK, Uebelhart D, Fransen J, Aufdemkampe $G$ (2005) Physical exercise in cancer patients during and after medical treatment: a systematic review of randomized and controlled clinical trials. J Clin Oncol 23: 3830-3842.

20. Dandapani SV, Sanda MG (2008) Measuring health-related quality of life consequences from primary treatment for early-stage prostate cancer. Semin Radiat Oncol 18: 67-72.

21. Akimoto T, Kumai $\mathrm{Y}$, Akama T, Hayashi E, Murakami $\mathrm{H}$, et al. (2003) Effects of 12 months of exercise training on salivary secretory $\lg \mathrm{A}$ levels in elderly subjects. $\mathrm{Br} \mathrm{J}$ Sports Med 37: 76-79.

22. Drela N, Kozdron E, Szczypiorski P (2004) Moderate exercise may attenuate some aspects of immunosenescence. BMC Geriatr 4: 8.

23. Foss FM (2002) Immunologic mechanisms of antitumor activity. Semin Oncol 29: 5-11.

24. Dieli-Conwright CM, Lee K, Kiwata JL (2016) Reducing the risk of breast cancer recurrence: an evaluation of the effects and mechanisms of diet and exercise. Curr Breast Cancer Rep 8: 139-150.

25. Braam KI, van der Torre $P$, Takken $T$, Veening MA, van Dulmen-den Broeder E, et al. (2013) Physical exercise training interventions for children and young adults during and after treatment for childhood cancer. Cochrane Database Syst Rev 4.

26. Irwin ML, Smith AW, McTiernan A, Ballard-Barbash R, Cronin $\mathrm{K}$, et al. (2008) Influence of pre-and postdiagnosis physical activity on mortality in breast cancer survivors: the health, eating, activity, and lifestyle study. J Clin Oncol 26: 3958-3964.

27. Söntgerath R, Eckert K (2015) Impairments of Lower Extremity Muscle Strength and Balance in Childhood Cancer Patients and Survivors: A Systematic Review. Pediatr Hematol Oncol 32: 585-612.

28. Galea V, Wright MJ, Barr RD (2004) Measurement of balance in survivors of acute lymphoblastic leukemia in childhood. Gait Posture 19: 1-10.

29. Schoch B, Konczak J, Dimitrova A, Gizewski ER, Wieland $R$, et al. (2006) Impact of surgery and adjuvant therapy on balance function in children and adolescents with cerebellar tumors. Neuropediatrics 37: 350-358.

30. Turner M, Gagnon D, Lagace M, Gagnon I (2013) Effect of treatment for paediatric cancers on balance: what do we know? A review of the evidence. Eur J Cancer Care (Engl) 22: 3-11.

31. Kurpiers N, Kersting UG (2017) The one-ski-method-effects of an alternative teaching approach on selected movement patterns in alpine skiing. Cogent Soc. Sci. 3: 1-11.

32. Clark RA, Bryant AL, Pua Y, McCrory P, Bennell K, et al. (2010) Validity and reliability of the Nintendo Wii Balance Board for assessment of standing balance. Gait Posture 31: 307-310.

33. Bland JM, Altman DG (1995) Multiple significance tests: the Bonferroni method. BMJ 310: 170

34. Keats MR, Culos-Reed SN (2008) A community-based physical activity program for adolescents with cancer (project TREK): program feasibility and preliminary findings. $J$ Pediatr Hematol Oncol 30: 272-280.
35. Tanir MK, Kuguoglu S (2013) Impact of exercise on lower activity levels in children with acute lymphoblastic leukemia: a randomized controlled trial from Turkey. Rehabil Nurs 38: 48-59.

36. Taskinen MH, Kurimo M, Kanerva J, Hovi L (2013) Physical performance of nontransplanted childhood ALL survivors is comparable to healthy controls. J Pediatr Hematol Oncol 35: 276-280.

37. Mock V, Pickett M, Ropka ME, Muscari Lin E, Stewart KJ, et al. (2001) Fatigue and quality of life outcomes of exercise during cancer treatment. Cancer Pract 9: 119-127.

38. Fiorillo A, Rinaldi M, Foggia L (2010) Gait analysis in children treated by surgery followed by adjuvant therapy for posterior fossa tumors. Acta Neurologica Belgica 110: 306-310.

39. Shumway-Cook A, Woollacott MH (2001) Motor control: Theory and practical applications. Williams \& Wilkins.

40. Staff NP, Grisold A, Grisold W, Windebank AJ (2017) Chemotherapy-Induced Peripheral Neuropathy: A Current Review. Ann Neurol 81: 772-781.

41. Konczak J, Schoch B, Dimitrova A, Gizewski E, Timmann D (2005) Functional recovery of children and adolescents after cerebellar tumour resection. Brain 128: 1428-1441.

42. Götte M, Kesting S, Winter C, Rosenbaum D, Boos J (2014) Comparison of self-reported physical activity in children and adolescents before and during cancer treatment. Pediatr Blood Cancer 61: 1023-1028.

43. Järvelä LS, Niinikoski H, Lähteenmäki PM, Heinonen OJ, Kapanen J, et al. (2010) Physical activity and fitness in adolescent and young adult long-term survivors of childhood acute lymphoblastic leukaemia. J Cancer Surviv 4: 339-345.

44. Tan SY, Poh BK, Chong HX, Ismail MN, Rahman J, et al. (2013) Physical activity of pediatric patients with acute leukemia undergoing induction or consolidation chemotherapy. Leuk Res 37: 14-20.

45. Wampler MA, Galantino ML, Huang S, Gilchrist LS, Marchese VG, et al. (2012) Physical activity among adult survivors of childhood lower-extremity sarcoma. J Cancer Surviv 6: 45-53.

46. Winter C, Müller C, Brandes M, Brinkmann A, Hoffmann C, et al. (2009) Level of activity in children undergoing cancer treatment. Pediatric Blood \& Cancer 53: 438-443.

47. Wojtyczek B, Pasławska M, Raschner C (2014) Changes in the balance performance of polish recreational skiers after seven days of alpine skiing. J Hum Kinet 44: 29-40.

48. Müller E, Gimpl M, Kirchner S, Kröll J, Jahnel R, et al. (2011) Salzburg Skiing for the Elderly Study: influence of alpine skiing on aerobic capacity, strength, power, and balance. Scand J Med Sci Sports 21: 9-22.

49. Narici M, Flueck M, Koesters A, Gimpl M, Reifberger A, et al. (2011) Skeletal muscle remodeling in response to alpine skiing training in older individuals. Scand J Med Sci Sports 21: 23-28.

50. Nasuti G, Temple VA (2010) The risks and benefits of snow sports for people with disabilities: a review of the literature. Int J Rehabil Res 33: 193-198.

51. Kurpiers N, McAlpine P, Kersting UG (2017) Predictors of falls in recreational snowboard jumping: An observational study. Injury 48: 2457-2460. 